\title{
LOS TRASTORNOS NEUROLOGICOS OBSERVADOS EN EL CURSO DEL TRATAMIENTO DE LA MENINGITIS TUBERCULOSA TEL NISO
}

\author{
Dr. MARIO VENEGAS YAÑEZ *
}

Nuestra tesis fué realizada bajo la direceión de los Drs. Eduard|Brissau y Marcel Naveau, jefe del servicio de tisiología infantil el primero y emihente colaborador, el segundo, de la clínica del Profesor Debré y representa la experiencia de siete años sobre la materia, obtenida por el Profesor Debré y sus colaboradores. Esta experiencia está basada en el estudio minucioso de 700 fichas de enfermos ingresados entre el $1^{\circ}$ de Enero de 1947 y el 15 de Octubre de 1953. En nuestro trabajo personal debimos revisar y considerar las nuevas observaciones de los niños hospitalizados durante el año 1953 y debimos también reconsidetar algunos aspectos planteados en la observación de conjunto de las manifestalciones neurológicas aparecidas durante el tratamiento de la meningitis tuberculosa del niño, ateniéndonos en todo momento a la cla-

* Del Servicio de Pediatria del "Hospital Defor. mes", de Valparaíso. Resumen di Tesis reajizada en 1a Clinica del Prof. Debri, Facultad de Medicina de París.

\section{INTRODUCCIÓN}

sificación e ideas del Profesor Debré y colaboradores. Ante la imposibilidad material de publicar íntegramente nuestro trajajo, nos limitaremos a la exposición de las nociones más fundamentales.

En la actualidad los trastornos neurológicos observados durante el curso evolutivo y tratamiento de la meningitis tuberculosa del niño adquieren una importancia fundamental desde el punto de vista djagnóstico, tratamiento precoz y pronóstico.

Etiologia. Se identifica este estudio con el de la meningitis y nos atendremos a las ideas fundamentales.

Intervención microbiana. La inoculación puede hacerse por vía sanguínea o indirectamente por bacilos provenientes de un tuberculoma cerebral y favorecida por un traumatismo craneano, modalidad excepcional.

Período de incidencia. La mayoría de los casos de meningitis tuberculosa sobrevienen en Ios tres meses siguientes a la 
primo-infección $(58 \%)$ y $20 \%$ entre el tercer y duodécimo mes (encuesta J. Raynaud).

Modalidades de contagio. La meningitis aparece en un número considerable de casos después de una contaminación masiva, generalmente familiar $(43 \%$ encuesta Dra. Cremieu Alcan; posteriormente otra encuesta reveló un porcentaje de $35 \%$ de los casos en el Servicio del Profesor Debré).

Vías de penetración. Se hace en la mayoría de los casos por vía bronquial siendo excepcional otra vía. Es también excepcional el contagio por el bacilo bovino.

Estreptomicino-resistencia. Después de 1950 se ha observado un aumento extraordinario de los casos de infección por bacilos estreptomicino-resistentes, contabilizándose hasta 9 en las estadísticas; anteriormente el Profesor Debré tenia sólo un caso.

Meningitis y primo-infección. Las encuestas establecen una incidencia de 1 meningitis por 300 primo-infecciones y dos principios fundamentales: $1^{\circ}$ Las invasiones tuberculosas con imagen de foco ganglio-pulmonar nitido se complican más a menudo de meningitis que la primo-infección sin imagen radiológica. $2^{\circ}$ Las invasiones tuberculosas con tuberculosis $\mathrm{mi}-$ liar aguda o sub-aguda son las que tienen el mayor riesgo de posibilidades de tuberculosis meníngea. El peligro es inminente cuando la tuberculosis miliar afecta la coroides. De 113 casos de tuberculosis miliar con tubérculos coroídeos, 78 meningitis $(69 \%)$. En 51 tuberculosis miliar sin tubérculos coroideos se han comprobado sólo 24 meningitis $(47 / \mathrm{r})$.

Meningitis y factores favorecientes. La meningitis es más frecuente en los niños más jóvenes: en los menores de 2 años el $72 \%$ de los casos estudiados y cuyo contagio ha sido comprobado; de 2 a 5 años $69 \%$ de los casos en las mismas condiciones; de 5 a 12 años $39 \%$; después de los 12 años $31 \%$. Estos porcentajes corrasponden a grupos diferentes separados según sus respectivas edades.

\section{PERÍDO INICIAL DE LA MENINGITIS TUBEACULOSA}

El comienzo de la meningitis tuberculosa es siempre preciso, pero precedido por un período prodrómico impreciso durante el cual el enfermo ya no se siente en sly estado de salud normal, presentan- do trastornos como cambios de carácter, astenia, enflaquecimiento. En este periodo de pródromos que dura de 2 a 3 semanas los signos neurológicos tienen una importancia de primer orden. El trastorno más frecuente es el cambio de carácter, presentandose aisladamente o acompañado de otros signos como trastornos vasomotores o de la sensibilidad, sensación de hormigueo, accesos de calor, etc., signos que podemos calificar de habituales del periodo prodrómico. Existe, además, otra clase de signos que se caracterizan por la singularidad de su sintomatología y el largo espacio de tiempo transcurrido entre su aparición y el comienzo de la meningitis, denominados signos no habituales del período prodrómico.

Signos neurológicos habituales. Cambio de carácter del niño que se pone gruñón, hostil, inestable; algias de diversas localizaciones: faciales, hépato-biliares, epigástricas, algias de tipo pseudo-apendicular, lumbo-ciáticas, todas de dificil interpretación en el niño.

Interpretación etiológica de estos signos. Varias razones justifican su etiología tuberculosa: $1^{0} \mathrm{Su}$ evolución simultánea con signos prodrómicos indiscutibles como cambios de carácter, astenia, enflaquecimiento. $2^{\circ}$ Su asociación con complicaciones neurológicas posteriores, no siempre comprobadas. $3^{9}$ Su desaparición con el tratamiento. 40 La ausencia definitiva de reaparición en caso de curación de la meningitis.

Formas neurológicas no habituales de la fase prodrómica. Se caracterizan por su gravedad y su larga evolución y puede tratarse de crisis convulsivas generalizadas o de tipo Bravais Jacksoniano, parálisis o trastornos de la sensibilidad. En Ia patogenia de estos accidentes se puede considerar la existencia de tubérculos corticales o de alteraciones arteriales que preceden a la meningitis.

Manifestaciones neurológicas del comienzo de la meningitis tuberculosa. Volvemos a encontrar en esta fase las algias ya señaladas en el período prodrómico. especialmente los dolores abdominales y las otalgias.

Dolores abdominales. Entre 262 enfermos, 18 han comenzado de esta manera (5\% más o menosi. Se han observado diagnósticos errados de apendicitis agudas a causa de esta sintomatología dolorosa. 
Otalgias. Son de difícil interpretación en el niño. En ciertos casos coexisten con una otitis pero en otros el enfermo no presenta ninguna modificación de los tímpanos.

Síndromes de déficit funcional en foco de la meningitis tuberculosa en la fase de comienzo. Se les observa con una frecuencia de $15,5 \%$. Su expresión clínica más frecuente la constituyen las parálisis y las crisis convulsivas encontrándose a menudo asociadas estas ultimas a síndromes hemiplégicos. Entre las parálisis son las de los nervios óculo-motores las más importantes, en orden de frecuencia, siendo las del VI par las más frecuentes $y$ menos, las del III par. En cuanto al significado pronóstico, son las parálisis oculares precoces que aparecen antes del diagnóstico o durante Ios primeros días del tratamiento las de rhejor pronóstico.

Convulsiones. Siguen en frecuencia a las parálisis oculares y se observan a menudo asociadas a las hemiplegias. Pueden presentarse también aisladamente como crisis Bravais Jacksonianas o crisis convulsivas generalizadas. Las hemiplegias se presentan en tercer orden de frecuencia $y$ traducen un compromiso más grave del encéfalo. El cuadro hemiplégico es precedido frecuentemente por una crisis de tipo Bravais Jacksoniano, sensorial, motora o sensitivo-motora. El déficit sensorial puede traducirse por adormecimiento, hormigueo o hiperestesia de la zona afectada por la hemiplegia. La hemiplegia puede presentarse también de manera brusca sin pródromos ni convulsiones asociadas de nimguna especie.

En la fase de comienzo de la meningitis tuberculosa hemos observado otros trastornos neurológicos más raros como, por ejemplo, trastornos de la palabra en Ios casos en que una hemiplegia derecha provoca una afasia. trastornos de la eseritura $y$ trastornos esfinterianos, en los casos en que se presentan otras complicaciones neurológicas más graves.

Comienzo por trastornos de la consciencia. Los trastornos precoces de la consciencia son raros en la meningitis tuberculosa y su comprobación constituve un test clínico fiel para el pronóstico. En la mayoría de los casos estos trastornos son mínimos y su evolución hacia la agravación oscurece simultáneamente el oronóstico de la meningitis tuberculosa. Estos se pueden agrupar en tres categorías: $1^{\circ}$ Los trastornos de la consciencia de la meningitis tuberculosa del lactante. $2^{\circ}$ Los estados de coma profundo que aparecen en la primera semana. $3^{\circ}$ Los trastornos progresivos precoces de la consciencia.

Los trastornos de la consciencia en el lactante presentan dificultades para su interpretación y traducen siempre un estado muy avanzado de la meningitis tuberculosa $\mathrm{y}$ un pronóstico severo. Los signos más frecuentemente observados son la somnolencia y la mirada fija.

Comas profundos y repentinos que aparecen en la primera semana están frecuentemente asociados a estados convulsivos o a cuadros hemiplégicos y traducen una forma gravísima de la enfermedad desde el comienzo.

Los trastornos progresivos precoces de la consciencia, son la modalidad observada con mayor frecuencia en el comienzo de la meningitis del niño, hacen temer otras complicaciones neurológicas y constituyen un índice clínico de primera importancia para apreciar la efícacia del tratamiento, sospechar la existencia de formas estreptomicino-resistentes o un compromiso muy severo del encéfalo.

Complicaciones neurológicas aparecidas durante la evolución de la meningitis tuberculosa. Son susceptibles de mcdificar el pronóstico de la enfermedad, ya sea porque traducen una agravación de la misma o una reactivación. Citaremos las más importantes.

19. Parálisis de los nervios óculo-motores. Tienen un mal pronóstico cuando aparecen después de los primeros quinee días de tratamiento. El carácter bilateral de ellas representa también otro elemento de mal pronóstico, observándose a menudo compromisos bilaterales del motor ocular externo; se atribuye a la hipertensión intracraneana el origen de este trastorno. En cuanto a localización, pueden considerarse dos tipos: parálisis intrinsecas y extrínsecas. Entre las intrínsecas mencionaremos las alteraciones pupilares. Entre 85 enfermos con meningitis bacilar 41 presentaban trastornos pupilares. Se ha observado 27 veces desigualdad pupilar (21 fallecimientos i, 11 veces midriasis paralítica simétrica (9 fallecidos), 2 veces miosis acentuada bilateral e igual ( 2 fallecimientos i y 17 veces arreflexia completa a la luz 115 fallecimientos. Estas cifras indican la gravedad pronóstica ajena a fecha de aparición del trastorno. 
Parálisis extrínsecas. En el mismo grupo anterior 28 casos presentaron parálisis oculo-motoras; 15 con parálisis bilateral del VI par (11 fallecieron y 4 mejoraron con regresión completa de la parálisis); otros 7 presentaron parálisis unilateral del VI par ( 5 fallecimientos y 2 mejorías con curación de la parálisis ); en un tercer grupo se comprobaron 6 casos con parálisis unilaterales del III par ( 3 totales y 3 parciales) y Ios 6 enfermos fallecieron. Śe comprobó, además, 4 veces, en pleno coma, una oftalmoplegia completa extrinseca e intrinseca preagónica que no se clasificó. En 4 casos una desviación conjugada de la cabeza y de los ojos ( 3 fallecimientos; el sobreviviente conserva secuelas graves de hemiplegia y una hemianopsia lateral homónima). En 6 enfermos se observó nistagmus espontáneo en el comienzo de la enfermedad meníngea 3 fallecieron $y$ en los 3 restantes desapareció); se trataba probablemente de un trastorno provocado por la inflamación. sin compromiso orgánico serio. La mayoría de las parálisis óculo-motoras son precoces y contemporáneas de la fase aguda regresando en uno o dos meses sin dejar secuelas. Lał parálisis oculares del período evolutivo son más raras, agravan seriamente el pronóstico de la enfermedad y en su mejoría se observan secuelas.

$2^{\circ}$ Hemiplegias en el curso de la meningitis tuberculosa. Podemos observar diferentes grados de gravedad en estas hemiplegias, pudiendo ser incompletas, parciales, las más frecuentes o hemiplegias masivas, completas $\mathrm{y}$ totales. Todas estas hemiplegias tienen siempre las características de las hempilegias corticales. Siempre los trastornos son más acentuados en el miembro superior que en el ínferior y las parálisis de la cara más impartantes aue las del miembro superior. Por lo demás, en algunos casos la parálisis facial constituye lo esencial por una paresia discreta y un signo de Babinski.

Patogenia. En la mayoría de las observaciones existen dos argumentos en favor del origen vascular de la hemiplegia: su marcha evolutiva y su aspecto clínico. La marcha evolutiva del trastorno es muy evocadora de un reblandecimiento por arteritis. Precedida de pródromos transitorios como parestesia, paresias discretas, afasia, la hemiplegia aparece bruscamente. El cuadro clínico puede aún completarse con hemianopsia, movimientos anor- males, correspondiendo a un territorio vascular conocido.

Pronóstico. Podemos decir que toda hemiplegia es un factor agravante del pronóstico de la meningitis tuberculosa y si no lo es siempre desde el punto de vista vital to es desde el aspecto funcional por las secuelas. La fecha de aparición de una hemiplegia es también un factor importante para el pronóstico, pudiéndose distinguir las de aparición precoz y las que aparecen tardiamente. Las hemiplegias precces se caracterizan por su pronóstico benigno a pesar de que a veces presenten caracteres de gravedad con sintomatología de hemiplegia total y completa. A pesar de ello y del estado del niño, después todo regresa quedando discretísimas secuelas; otras veces la hemiplegia es parcial y no hay secuelas.

Hemiplegias tardías. En principio tienen mal pronóstico, ya sea porque aparecen durante un tratamiento mal llevado o porque traducen formas de evolución de meningitis a bacilos resistentes al tratamiento. Su gravedad se debe a la evolución de las lesiones tuberculosas que ellas señalan. Como en las formas precoces, encontramos aqui también un acentuado polimorfismo del síndrome y la asociación a otras complicaciones neurológicas de los más diversos caracteres y gravedad.

Crisis convulsivas. Se observan precediendo a la hemiplegia o acompañándola en su evolución, ya sea como crisis Bravais Jacksonianas o como crisis epilépticas generaljzadas, pudiendo estas últimas suceder a crisis localizadas o alternar con ellas, aún dependiendo de un foco, como lo atestigua la hemiplegia o la localización electro-encefálica. Tienen también un pronóstico más sombrío cuando aparecen en plena evolución de la meningitis y no en su comienzo. Se distinguen muy especialmente por su gravedad las convulsiones que aparecen complicando formas de evolución gxave de la enfermedad.

Hemicoreas. Se consideran también como un sindrome de déficit en foco. Tomando en cuenta los trastornos vasculares como causa, debemos comprender que ellos comprometen una región más extensa y muy especialmente los núcleos caudados del cuerpo estriado y su vecindad, pudiendo también afectar el cortex, la piamadre y la aracnoides. Pueden ser precoces y tardías, estas últimas, de pronóstico más sombrio, aparecen después del 
décimo quinto día de la enfermedad. Pueden presentarse aisladamente o asociadas a una hemiplegia y quedar como secuelas una vez mejorada esta.

Crisis tónicas posteriores. Son provocadas por lesiones irritantes de los núcleos intracerebelosos, adquieren caracteres exclusivamente tónicos y reproducen transitoriamente la actitud de la rigidez de descerebración. Jackson las ha descrito en los tumores del vermis cerebeloso. Traducen casi siempre una hipertensión intensa de la fosa posterior y representan un signo de sufrimiento del tronco cerebral. Su aparición hace temer un encajamiento de este. En presencia de una de cstas crisis $y$ de signos evidentes de hipertensión intracraneana como distensión de las suturas, edema papilar, trastornos de la consciencia más o menos pronunciado, se debe intervenir de urgencia por trépano-punción ventricular, isuspendiendo aI mismo tiempo las punciones lumbares. Estas crisis se presentan raramente en forma aislada y constituyen frecuentemente un episodio premonitorio de I una actitud de descerebración permanente. Por su patogenia constituyen un signo de mal pronóstico en el curso de la mentingitis tuberculosa.

COMPLICACIONES NEUROLÓdICAS APARECIDAS EN LA FORMA PROLONGADA DE LA MENINGITIS TUBERCULOSA

Los trastornos neurológicos que se presentan en el curso evolutivo de una meningitis tuberculosa crónica constituyen un capítulo totalmente nulevo de la tuberculosis e impulsan al médico a buscar los medios terapéuticos más diversos y audaces. A pesar de que no nos corresponde describir las características de la meningitis tuberculosa crónich recordaremos suscintamente las condicibnes de su constitución. Estas diversas condiciones se asocian muchas veces para originar esta forma de evolución crónica y caprichosa. Esta forma puede evolucionar bajo tres circunstancias diversas :

$1^{4}$ Por un tratamient $\phi$ insuficiente de la meningitis en su comienzo.

$2^{\circ}$ Por un diagnósticol tardío.

$3^{\text {\% }}$ Por cierta resistencia del bacilo a los medicamentos empleados.

Estas tres condiciones realizan estados de equilibrio entre la tendencia evolutiva de la enfermedad y la actividad del tra- tamiento. Las complicaciones neurologicas en esta forma de la meningitis son muy variadas pudiéndose observar síndromes semejantes a los descritos precedentemente $\mathbf{u}$ otros típicos a este período, que consideraremos.

Cisternitis posteriores. Representan una de las formas anátomo-clínicas más interesantes y graves de la meningitis tuberculosa. Es conocida la predilección de la tuberculosis por los plexos coroideos de la base del encéfalo. La aracnoiditis que estas lesiones producen determinan importantes y graves perturbaciones de la circulación del líquido céfalo-raquídeo y como una consecuencia la hidrocefalia. Recordaremos someramente que estas lesiones pasan por tres etapas: $1^{\text {a }}$ aracnoiditis congestiva, hiperhémica, inflamatoria; $2^{\text {a }}$ aracnoiditis con lesiones tuberculosas específicas, infiltrados, folículos, granulaciones; $3^{a}$ aracnoiditís fibrocaseosa. Esta aracnoiditis determina por una parte in bloqueo basal con hidrocefalia y, por otra, la alteración de los vasos que la atraviesan y que irrigan el diencéfalo y los núcleos estriados. La prueba de la encefalografía muestra que no hay ya circulación posible entre el cuarto ventriculo y los espacios subyacentes. Es sobre todo en el cadáver donde se observa más claramente este tjpo de lesiones. En efecto, el estudio anátomo-patológico informa respecto a la predilección de las lesiones por las meninges de los lagos de la base, especialmente, de la cisternitis interpeduncular y opto-quiasmática. Después, las lesiones, partiendo de la región opto-quiasmática, invaden progresivamente la región bulbar y cerebelosa. Sin embargo, no parece que la evolución de las lesiones de aracnoiditis basal sean obligatoriamente fatales. En muchos casos gracias a la intensificación del tratamiento antibiótico y al empleo de estreptomicina por vía sub-occipital y ventricular puede obtenerse la curación; en otros casos, desgraciadamente, las lesiones continúan su marcha progresiva a pesar del tratamiento.

Los signos clínicos son siempre de aparición tardía y a veces engañosos. Hay que tomar en cuenta, sin embargo, ciertos sintomas como signos vegetativos menores, intermitentes: temblor discreto y ligera hipertonia. Estos signos traducen un comienzo de compromiso de los centros diencefálicos y estriados, de naturaleza vascular muy probable. Los signos del líqui- 
do céfalo-raquideo son más importantes. Así, por ejemplo, la comprobación de un líquido ventricular normal o casi normal $\mathrm{y}$ de un liquido sub-occipital muy perturbado, con aumento progresivo de la albúmina, constituye un importante argumento en favor de la formación de una aracnoiditis basal.

Formas espinales de la meningitis tuberculosa. Entre 114 enfermos tratados on el servicio antituberculoso del Hospital "Enfants Malades" hasta el 1 ' de Enero de 1952 los sindromes correspondientes a una aracnoiditis espinal eran muy raros. En Montpellier, Janbon y sus colaboradores han publicado un trabajo en que consideran la existencia de aracnoditis espinal en el $45 \%$ de los casos. Debré, Brissaud y sus colaboradores se preguntan si estas lesiones son más frecuentes en las formas del adulto que en las del niño. En el niño se observan dos modalidades clínicas: parálisis periféricas por compresión radicular y paraplegias espasmódicas por compresión medular. En el adulto se han descrito también aracnoiditis asociadas a un síndrome de mielitis transversa.

El estudio de la circulación en el canal raquídeo muestra una hipotensión del líquido y la ausencia de cambios de la presión por la maniobra de QueckenstedStockey, signos que indican un bloqueo. E1 líquido muestra siempre una hiperalbuminosis, pero esta puede depender de un trastorno de la circulación espinal o puede ser debida a fenómenos más complejos tales como trastornos de la secreción y de la reabsorción, hemorragias meníngeas e hiperalbuminosis difusa, dado que es normal encontrar en la región lumbar, durante la evolución de la meningitis, un líquido con 4 o 5 grs. de albúmina, mientras que las cifras en el ventrículo raramente sobrepasan de 0,00 a 1 gr. en cl mismo enfermo.

Compresiones radiculares. Se observaron tres casos. En dos se trataba de una jaraplegia flácida, periférica, cuya sintomatología y exámenes eléctrjcos eran comparables. En uno de ellos los trastornos aparecieron en el primer mes de la enfermedad $y$ en el atro con ocasión de una recaída. Este sindrome radicular se caracteriza por dolores violentos, intolerables, atrofia $y$ enfriamiento de los miembros, hiperestesia al tacto y signo de Lasègue generalmente bilateral. En am- bos la evolución del cuadro se hizo progresiva hasta llevar al enfermo al coma. En el tercer caso, el cuadro evoluciona hacia la mejoría después de diez meses de enfermedad.

Compresiones medulares. Pueden ser localizadas o difusas. Etienne Bernard observó un caso indiscutible de síndrome radicular característico con signos cordonales subyacentes, que atestiguaban la existencia de una lesión localizada. El cirujano practicó una laminectomía y la ablación de un verdadero rodete aracnoídeo, lo que permitió una recuperación total.

Compresión difusa. En esta modalidad los signos semiológicos van precedidos casi siempre por trastornos esfinterianos $y$ algias radiculares mal localizadas. En estos casos la paraplegia espástica o flácidoespastica motriz pura, de evolución lenta - sub-aguda, es a menudo la primera etapa de una mielitis transversa; otras veces el síndrome conserva su autonomía semiológica. Se observó un síndrome paraplégico espasmódico determinado por una verdadera paquimeningitis caseosa comprobada en la autopsia.

Síndromes parkinsonianos. Representan una forma de evolución prolongada de meningitis tuberculosa con lesiones encefálicas pronunciadas. Como características fundamentales citaremos: temblores, rigidez, bradicinesia, conservación de las actitudes, como expresión de sufrimiento de los núcleos grises centrales, sufrimiento explicable por la hipertensión ventricular crónica. Se observa, además, los signos comunes a todas las formas de evolución crónica como: incontinencia esfinteriana, clonus inagotable de los pies, trastornos de la conciencia (obnubilación, hipersomnolencia o bradipsiquia).

Formas con rigidez de descerebración. Este sindrome se observa en los niños en los cuales la enfermedad meníngea ha determinado lesiones de extrema gravedad. Estas formas de meningitis crónica tienen los caracteres siguientes: existe desde un comienzo una hipertensión intracraneana considerable, la hipertensión determina primero un trismus, una pronación permanente de los antebrazos con los puños cerrados, incontinencia de los esfínteres, trastornos de la consciencia, tremulación y clonus inagotable de los pies. Después se instala la gran contractura, dolorosa e 
irreductible, y que se exagera a menudo durante los accesos convulsivos o hipertónicos exclusivos. Esta irigidez no realiza en todos los casos las mismas actitudes; evoca a veces la de la descerebración experimental, con los cuatro miembros en extensión, manos en prohación, puños cerrados, nuca y tronco encurvados en arco. Se pueden observar asociados fenómenos de adducción o abducción de los miembros, de rotación de la cabeza y de inclinación lateral del tronco. Existe otra actitud con mayor semejanza a la provocada por los tumores voluminosos de la base; en ella se observa una flexión de los miembros superiores asolciada a la extensión de los niembros inferiores. Estas dos actitudes pueden ser observadas alternativamente en el mismo enfermo y la flexión puede alcanzar un grado variable aún en los miembros inferiores. Se pueden observar asi todos los aspectos intermediarios entre la gran actitud de descerebración y la hiperflextión de los miembros de las formas de "meningitis prolongadas caquectizantes".

Las formas evolutivas de la meningitis tuberculosa con rigidez de descerebración representan una de las imodalidades más graves de la meningitis crónica. Su gravedad está asociada a una hipertensión permanente que produce un sufrimiento profundo de todas las vías de regulación del tonus y de los centros corticales. La dilatación monstruosa de los ventrículos que se observa en estos casas y la compresión brutal ejercida: sobre la corteza es una demostración de la importancia de los trastornos que provoca esta hipertensión permanente y crónica. La aparición de esta actitud parece ser favorecida por la poca edad del niño, por debajo de los dos años, más lenta para constituirse en el niño de más edad yl excepcional después de los seis años, no observándose en la fase terminal.

Formas con contractura en flexión. Este sindrome ha sido descrito anteriormente en el curso evolutivo de la meningitis por meningococos (meningitis prolongadas caquectizantes). En esta actitud la rigidez es acompañada de una flexión de los cuatro miembros. Esta contractura en flexión puede ser secundaria y reemplazar una rigidez de descerebración sin modificar el pronóstico de la enfermedad meníngea. En los casos que consideramos esta actitud es primitiva y en ningún momento se ha observado la menor contractura en extensión. Estas formas tienen interés por el hecho de que, a pesar de su gravedad, su pronóstico no es desesperado como se habia señalado en otro tiempo en las meningitis cerebro-espinales. Poseemos dos observaciones, una de las cuales evolucionó hasta la curación definitiva $y$, la otra, la dejamos en estado estacicnario y con posibilidades de recuperación progresiva. Consideramos que este síndrome está ante todo bajo la dependencia de la hiperestesia y secundariamente esta actitud se hace permanente por las retracciones tendinosas. Estas actitudes ceden, por lo demás, notabJemente con la movilización pasiva $\mathrm{y}$ eventualmente con las intervenciones quirúrgicas de alargamiento de los tendones, después de la curación de la meningitis. Es indispensable también, para asegurar la curación, una nutrición suficiente y el suministro de agua, calorias, proteinas y vitaminas necesarias.

Hemorragias meníngeas. Estas pueden manifestarse en cualquier período de la enfermedad. Nos atendremos a su estudio clínico suscinto sin considerar su compleja patogenia. Se observan bajo tres aspectos principales: I" Las hemorragias recidivantes en cada raquicentesis; $2^{\circ}$ las hemorragias abundantes intracraneanas; $y$ $3^{0}$ los hematomas sub-durales.

Hemorragias recidivantes. Pueden producirse por una predisposición a la hemorragia y aparecer en cualquier momento de la enfermedad. Esta tendencia hemorrágica existe en todos los enfermos en tratamiento por el PAS y se trata de combatirla con la inyección semanal sistemática de vitamina $K$. Se traduce por un signo constante: líquido sanguinolento en las raquicentesis o las punciones ventriculares, signo que puede ir asociado a otros más discutibles como la contractura del raquis y de los muslos, vómitos, anorexia, pérdida de peso, ete.

Hemorragias abundantes intracraneanas. Se han observado dos clases de accidentes: la inundación ventricular y el hematoma peribulbar voluminoso.

Derrames sanguíneos sub-durales. La existencia de un hematoma sub-dural era considerado en relación probable con un traumatismo local y se ha observado muy raramente este accidente grave en los ni- 
ños tratados con punciones ventriculares. Puede producirse, sin embargo, espontáneamente, durante la evolución de la meningitis tuberculosa $y$ su sintomatología brutal debe permitir sospecharlo en los cascs en que se ha podido comprobar una tendencia hemorrágica anterior. Esta sintomatologia violenta y brusca se traduce especialmente por colapso con pérdida de la consciencia y lipotimia y que muchas veces contrasta con el estado de relativa benignidad anterior del paciente.

Ceguera. No podriamos dejar de considerar, en este resumen, esta complicación gravísima. En cierto número de casos, en ausencia de una evolución grave de la meningitis, aparece una disminución de la agudeza visual sin edemas ni estasis de la papila. A veces ésta tiene un aspecto blanquecino, sin brillo, con vasos finos. Estos signos deben hacer temer la aparición de una atrofia óptica. En este caso, una intervención quirúgica permite siempre descubrir sobre el quiasma y los nervios ópticos, a veces, falsas membranas espesas que envuelven los nervios, estando los mismos cubiertos de tubérculos o un manto anacnoídeo sin tubérculos o verdaderos tuberculomas que comprimen los nervios y que es necesario disecar. La causa de la ceguera es, entonces, evidente $y$ la intervención va casi siempre seguida de una recuperación total de la visión. En otros casos la disminución de la agudeza visual es descubierta en niños con meningitis muy graves, con signos evidentes de hipertensión intracraneana, estasis papilar o distensión franca de las suturas del cráneo con reflejos foto-motores muy débiles o abolidos. En estos enfermos la exploración operatoria del quiasma y de los nervios ópticos permite comprobar las mismas lesiones anteriores. Sin embargo, es probable que estos niños no recuperen una visión normal; muchos de ellos mueren durante el curso de la reactivación evolutiva de la meningitis $\mathrm{y}$ otros quedan ciegos. Son todos niños con trastornos graves de la consciencia y estado general precario. Por último, en ciertos casos puede existir un estasis papilar bilateral o unilateral asociado a una disminución de la agudeza visual y sin trastorno grave de la consciencia. En estos casos la liberación quirúrgica de los nervios ópticos da-mejores resultados.

\section{PRONÓSTICO}

Nos limitaremos a considerar algunas ideas generales que deben tenerse siempre presente para la consideración del pronóstico de estos trastornos neurológicos.

Edad. Las manifestaciones neurológicas evolucionan tanto más fácilmente hacia la agravación progresiva cuanto más joven es el niño. Existen dos razones: 19 los accidentes asfícticos que se presentan en los niños muy jóvenes durante el tratamiento; y 2" las reactivaciones y las recaídas más frecuentes, especialmente estas últimas. En sí misma la meningitis no tiene ninguna característica especial. Se observan, sin embargo, algunos hechos paradojales. En el lactante la miliar, a pesar de los accidentes asficticos, es un factor de buen pronóstico pues su comprobación permite a menudo un diagnóstico precoz. Así, también, sus posibilidades de recuperación ante una lesión en foco es muy grande y esta posibilidad es menor en los de más edad.

Calidad del contagio. A este respecto hay que considerar si el contagio ha sido masivo o episódico, lo que es bien difícil de probar en la mayor parte de los casos. Debe tomarse en cuenta también la intervención de un bacilo estreptomicino-resistente lo que impide actuar eficientemente en el tratamiento.

Existencia de una diseminación miliar visible. En estos casos la mortalidad asciende hasta $61 \%$, siendo la mortalidad media global de 45 , tratamiento con estreptomicina y PAS i.

Lesión meningea. Un factor primordial del pronóstico es la amplitud de las lesiones meníngeas en el momento en que se instituye el tratamiento. La precocidad del tratamiento condiciona en forma indiscutible su eficacia.

Estado del enfermo en el momento de prescribir el tratamiento. En la noción del estado del niño hay que considerar varios factores: el trastorno de la consciencia, el electro-encefalograma y los caracteres del liquido céfalo-raquideo.

Trastorno de la consciencia. Se distinguen, en el comienzo de la meningitis tuberculosa, cinco estados diferentes de la consciencia: $1^{\circ}$ Consciencia normal $2^{\circ} \mathrm{Ob}$ nubilación. Trastorno discreto caracteri- 
zado por hostilidad o indiferencia, cierta fatiga intelectual y lentitud de las respuestas. 3" Somnolencia $p$ hipersomnolencia simple que se agrega a uno de los estados precedentes. Es fácil despertar al enfermo que permanece lúcido mientras se preocupan de él peró que se duerme nuevamente en cuanto parece que se le abandona. 4? Torpor. Trestorno profundo $y$ permanente en que el enfermo sigue todavía con la mirada, reconoce a sus padres, responde sumariamente a las preguntas y ejecuta una orden simple. $5^{\circ} \mathrm{Co}-$ ma. Interrupción completa de la vida de relación. Según las estadísticas del Profesor Debré y colaboradores la mortalidad pasa de $17 \%$ a $89 \%$ en los casos extremos, es de 30 a $34 ;$ en los casos de obnubilación o somnolencia y sube a $68 \%$ cuando el enfermo se sume en el torpor.

Electro-encefalograma, Se distinguen 5 tipos de trazados en la /meningitis tuberculosa del niño: El tipo! $N$ corresponde al electro-encefalograma normal, tomando en cuenta la edad del niño. El tipo 1 , en el cual el ritmo de base no está modificado, pero muestra algunas ondas aisladas y raras. El tipo II conserva todavía un ritmo de base normal y claro. Sin embargo, a intervalos aparecen accidentes importantes, verdadercs trozos de ondas lentas, sincrónicas, de duración variable, tomando después el trazado su ritmo normal. En el tipo III casil no se ve trazado de ritmo de base. Las ondas lentas apare cen en forma casi ininterrumpida, conservando siempre cierta regularidad. El trazado de tipo IV es absolutamente anárquico pues no solamente el ritmo de base ha desaparecido totalmente sino que, además, las ondas lentas son de forma, amplitud y ritmo muy variables.

El trazado eléctrico permite repartir a los niños en tres grupos cuya mortalidad es muy diferente. Estal es de $18 \%$ si el trazado es normal o polco alterado (tipos I y II), de $49 \%$ cuando los trastornos eléctricos son más severos (tipo III) y alcanza un $71 \%$ cuando el electro-encefalograma es completamente anárquics (tipo IV). Pero estas cifras dan solamente una idea muy general y es preciso hacer exámenes electro-encefalográficos repetidos para obtener una información pronóstica más, valedera y además hacer comparaciones con el estado de iconsciencia del nino que permite corregir en muchos casos un pronóstico demasiado pesimista.
Los caracteres del líquido céfalo-raquídeo. Dado que las alteraciones del líquido céfalo-raquídeo se establecen en forma progresiva hasta la intervención del tratamiento, su importancia está en relación con el carácter más o menos tardío del diagnóstico. En sintesis, podemos recalcar que el pronóstico de las manifestaciones neurológicas de la meningitis tuberculosa está bajo la dependencia del pronóstico de la enfermedad meningea misma.

Pronóstico desde el punto de vista funcional. Es benigno para Jas manifestaciones neurológicas aparecidas en la fase prodrómica o al comienzo de la enfermedad c durante los primeros quince días del tratamiento antituberculoso $\mathrm{y}$ en la mayoría de estos casos se observa una regresión casi completa de los trastornos. Diferente es el pronóstico si las manifestacio nes neurológicas aparecen después de los primeros quince días del tratamiento o más tardiamente en relación con una recaida o una reactivación de la enfermedad. En estos casos se pueden observar todavía regresiones completas de los trastornos con la curación de la meningitis, pero la persistencia de las secuelas es más probable y estas secuelas son tanto más graves cuanto más larga ha sido la evolución de la enfermedad.

\section{TRATAMIENTO}

El tratamiento de las manifestaciones neurológicas de la meningitis tuberculosa se identifica con el de la meningitis misma, salvo las indicaciones muy particulares que exigen ciertos trastornos en estado de secuelas.

Según la experiencia de estos últimos años se considera la estreptomicina como la base del tratamiento con la asociación actualmente de PAS e INH (isonicotinil hidrazida) por vía general y local. Para obtener los mejores resultados hay que contar con una organización metódica para la vigilancia de los niños enfermos. Esta vigilancia sistemática comprende los diferentes medios de investigación y control más indispensables, a saber: $1^{\circ}$ exámenes clínicos regulares con un examen neurológico periódico y búsqueda minuciosa de los signos de localización tuberculosa; $2^{\prime}$ exámenes pur lo menos semanales de líquido céfalo-raquídeo y cotidianos en caso necesario debiendo comprender el recuento celular, el porcentaje de 
albúmina, de glucosa y cloruros y de sus respectivas curvas; $3^{\circ}$ investigación repetida del bacilo de Koch en el líquido céfalo-raquídeo, en los cultivos y las inoeulaciones al cobayo y el estudio de la sensibilidad del germen a los diferentes medicamentos tuberculosos; $4^{\circ}$ examen oftalmológico sistemático para estar informa. do constantemente sobre la evolución de los tubérculos coró́deos bajo la influencia del tratamiento y además del estado de la papila, del edema y estasis papilar que pueden determinar una conducta terapéutica especial en el comienzo o durante la evolución de la enfermedad: 5? la vigilancia de la función auditiva del enfermo es indispensable para apreciar precozmente una disminución de la agudeza auditiva y modificar la dosis de estreptomicina; y $6^{*}$ las indicaciones de los trazados electro-encefalográficos suministran informaciones indispensables para apreciar correctamente la evolución de la enfermedad meníngea.

No consideramos, por ahora, necesario extendernos en lo que respecta al tratamiento médico propiamente tal, dada la tendencia actual por parte de varios autores del abandono de la via intratecal en la administración de los medicamentos antituberculosos, tendencia que es considerada todavía en su etapa inicial y con reducido material de prueba, por otros, como para ser aceptada en forma sistemática y definitiva, en todos los casos.

\section{CONCLUSIONES}

1. Las manifestaciones del periodo prodrómico de la meningitis tuberculosa tienen una importancia fundamental para el diagnóstico de la enfermedad meníngea.

2. Los trastornos neurológicos aparecidos durante el tratamiento traducen la importancia del compromiso meningo-encefálico.

3. Entre los trastornos neurológicos más importantes hay que considerar las parálisis oculares, las crisis convulsivas, las hemiplegias, las cisternitis posteriores, Ia ceguera y las formas espinales.

4. Las complicaciones neurológicas agravan el pronóstico vital de la meningitis tuberculosa y su gravedad está en relación con la fecha de aparición de estos trastornos durante el tratamiento. Las manifestaciones neurológicas aparecidas antes del décimo-quinto día del tratamiento tienen un pronóstico más benigno tanto desde el punto de vista funcional como desde el punto de vista vital.

5. La meningitis tuberculcsa tratada ha mostrado sindromes muy raramente observados anteriormente y debidos a la evolución crónica de las lesiones tuberculosas meningo-encefálicas.

6. Entre las manifestaciones neurológicas de la forma crónica de la enfermedad hay que señalar los síndromes parkinsonianos, las formas con rigidez de descerebración, las con contractura en flexión y las con hidrocefalia.

7. El pronóstico funcional y vital de estas formas es más grave en relación con la gravedad de la enfermedad meníngea y son las formas con rigidez de descerebración y las con hidrocefalia las que representan los casos de pronóstico desesperado.

8. El trastorno neurológico más grave, desde el punto de vista funcional, es la ceguera, trastorno que puede ser mejorado con una intervención neuro-quirúrgica oportuna sobre la región opto-quiasmática.

9. Entre las complicaciones neurológicas aparecidas durante la evolución de la meningitis tuberculosa tratada. las más temibles, desde el punto de vista vital, son las hemorragias meningeas y muy especialmente las hemorragias abundantes intracraneanas y los derrames sanguíneos subdurales que exigen una intervención quirúrgica inmediata.

10. El pronóstico vital de todas las manifestaciones neurológicas de la meningitis tuberculosa se identifica con el de la enfermedad meníngea y está subordinado a todos los factores de este último. Entre estos la edad es un elemento importante de gravedad.

11. El tratamiento de todos estos trastornos está subordinado a la terapéutica de la meningitis tuberculosa en cada caso particular.

12. Las indicaciones más importantes del tratamiento neuro-quirúrgico son: hipertensión intracraneana, hidrocefalia, ceguera y hemorragias intracraneanas.

13) Las secuelas de los trastornos neurológicos de la meningitis tuberculosa requieren además un tratamiento de recuperación funcional por los diversos métodos de reeducación y readaptación de que dispone la terapéutica médica. 


\section{SUMMARY}

700 patients with tuberculous meningitis treated by Prof. R. Debré and coworkers are analyzed within the years 1947-53. The study is centered in the neurologic disorders appeared during the treatment of the disease and in relation to which it is interesting to point out the following:

Among the most important neurologic disorders are: ocular paralysis, convulsions, hemiplegia, posterior cisternitis, blindness and spynal forms. These complications aggravate the vital prognosis of the tuberculous meningitis.

The tuberculous meningitis treated has shown syndrome very rarely observed before and due to the cronic evolution of the meningoencephalitic lesions. Among the neurologic disturbances of the cronic form the following should be point out: the parkinsonian syndromes, the forms with rigidity of decerebration, the ones with contractures in flexion and the ones with hydrocephalus. The functional and vital prognosis of these complications is bad and the ones with the worst prognosis are those of rigidity of decerebration and those with hydrocephalus. From the functional point of view the most serious complication is blindness, which in certain cases can be cured by means of an opportune operation over the optochiasmatic region. At the same time, from the vital point of view, the most severe complications are the meningeal hemorrhages.

The most important indications for neurosurgical treatment are intracranial hypertension, hydrocephalus, blindness and intracranial hemorrhages. 